\title{
The Intelligent Home System Controlled by SMS ${ }^{*}$
}

\author{
ZHANG Jia-bo, BAO Ke-yu, LIU Xiang, FENG Chang-chun \\ School of communication and information engineering, Chongqing University of Posts and Telecommunications, Chongqing \\ 400065, China. \\ zhangjb@cqupt.edu.cn, 303098529@qq.com, toliuxiang@foxmail.com
}

\begin{abstract}
It includes two parts for the controlling system of intelligent home. The remote controlling part is a smart phone with Android apps, and another is the operation and execution part, which is a monitoring system controlled by the Single Chip Microcomputer (SCM). The later provides information collection, process and controlling, including SMS module of GSM — TC35i, relays and sensor devices.

Using encrypted short message commands through Android application software of intelligent mobile phone, you can easily implement wireless remote monitoring for intelligent home system connected with remote operation. The whole system is designed simply, and information transmitted security and reliability. The remote controlling system in intelligent home maybe cost the lowest, and expands the scope of application of the Internet of things.
\end{abstract}

Index Terms - Android apps, wireless monitoring, Short Message Services (SMS), intelligent home.

\section{Summarize}

With the rapid development of economy and the continuous improvement of our living standards, the intelligent home has become a part of our houses gradually. Intelligent home can provide the comfortable living conditions, which installed amount of intelligent devices. With residential platform, the system uses both wired and wireless network platform communication technologies, including integrated wiring systems, security systems, background music / broadcasting systems, lighting and curtain controlling systems, air-conditioning ones, as well as home theater ones. Intelligent home integrates all of the facilities relating to human's life in order to build efficient residential facilities and family schedules affairs management system. It can improve home safety, convenience, comfort, artistry, and can make an energy conservation and environmental protection living environment.

For its wide range of area cover, easy applications, the lower cost of building and compatibility, wireless network has a broad application prospects in the intelligent home [1]. Intelligent home products basing on wireless network access to market constantly. However, these products have a lot disadvantages such as too expensive equipment, poor reliability, easy to leak information, poor real-time capability and so on [2]. These are obstacles of market promotion.

Mobile telecommunication network is a digital mobile communications network. It has the most mature technology and the most extensive coverage of area. Transmitting the communication of the monitor information has a very prominent advantage with the high reliability. Particularly the SMS is easy to operate, and it has the abilities of long distance controlling and lower error rate and cost [3]. Meanwhile, with the popularity of smart phones and the adoption of Android development platform, Android monitoring software basing on SMS function will be favored by many users, and the market prospect is broad. This article details the design and implementation of this important application process.

\section{II . Systematic design}

Intelligent home system based on the short message module of GSM network and Android application software. The system consists of four parts, the remote control unit (equipped with a self-developed Android application software for mobile phones), the main control unit (89C51 microcontroller), wireless communication unit (the TC35i SMS module [4]), and the action execution unit (LCD display, relay, and a variety of sensor devices). As shown in Figure 1, the system is an open SMS module-based architecture of the intelligent home system, with strong expansion capability.

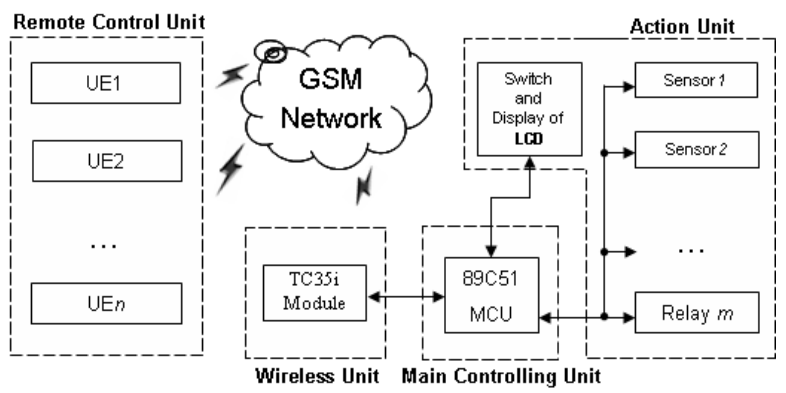

Fig. 1 Overall framework of controlling system

For instance, when those sensor interfaces of action unit were connected to the temperature, smoke, pressure sensors, these functions can be realized, such remote temperature measurement, fire alarms and anti-theft[5]. On the other hand, when those relays link with action units like conditioning(or lighting) switches, video camera switches and entrance guard switches, Etc., we can also achieve the same remote controlling functions. At the same time, the system includes LCD screen and the corresponding controlling switch, so users

\footnotetext{
* The National Key Scientific Instrument and Equipment Development Project of China(2012YQ20022404), The Student Research Training Project of cqupt(A2012-67)
} 
can extract stored manipulation data off-line, and make aided analysis.

There are too many other hardware design papers about wireless remote control system [6-9], so this article only makes opening hardware architecture, and focuses on the software design ideas and techniques. The paper introduce the monitoring of household air-conditions as an example of application. We can start or stop the air conditioner, and measure its temperature, and even adjust it through the relay and temperature sensor.

The sensor is an important detection apparatus. There are all kind of sensors [10], and their basic principles are same substantially. The sensor detects information and changes into electrical signals according to certain rules, then output. All of the gathered information can be transmited, storage, displayed and used.

When the host considers the need of opening his/her aircondition at home in advance on the way home, he/she only need to send a text message to the home operating execution unit through monitoring software of the phone, and the temperature sensor will capture the room temperature and return to $\mathrm{him} / \mathrm{her}$. If he/she decided to turn on the air condition, as long as he/she send a command to the appropriate relay, the air conditioning can be opened immediately. Command information was transmited Between Single Chip Microcomputer (SCM) and smart phone microcontroller through the TC35i module.

SCM sends encrypted messages to users with the AT command. After the user receiving the message, it needs to reply to a confirmed short message in order to improve the reliability of the communication. The entire design is simple, high reliability and low cost. Except to the TC35i, the system has no special chip. TC35i is compatible with TC35, and it can support Chinese SMS. TC35i has low power consumption, small size, and low price.

\section{III . Control software development}

Using a mobile phone as a remote control terminal, users can real-time control status of the execution unit by operating the Android applications. He can send query information, so that the execution unit will return reports of system running status and monitoring information. And also, he can send controlling commands directly, and can start the appropriate sensor or relay to perform the action. These operations are only required to obtain an operating license. In order to make users' information more secure and reliable, the system uses a four-layer security methods: provide the administrator's password when the user logins; input the authority password when executing controlling command; encrypt the sending messages and add the legitimate users' phone numbers to the white list. When the user sends the first command, the phone number will check with the white list.

\section{A . The design of $U I$}

UI is the abbreviation of User Interface. The UI design refers to the overall design of software, including humancomputer interaction, operating logic and beautiful interface.
Like the design of web page, layout of android's UI design bases on XML. UI is designed to shield the operation of the SMS completely, and with sound warning. By clicking a few buttons, the user is able to achieve control simply. All of these makes users have a better experience. Inspired by the "nine squares", the software interface of the monitoring system uses an intuitive interface, shown in Figure 2.

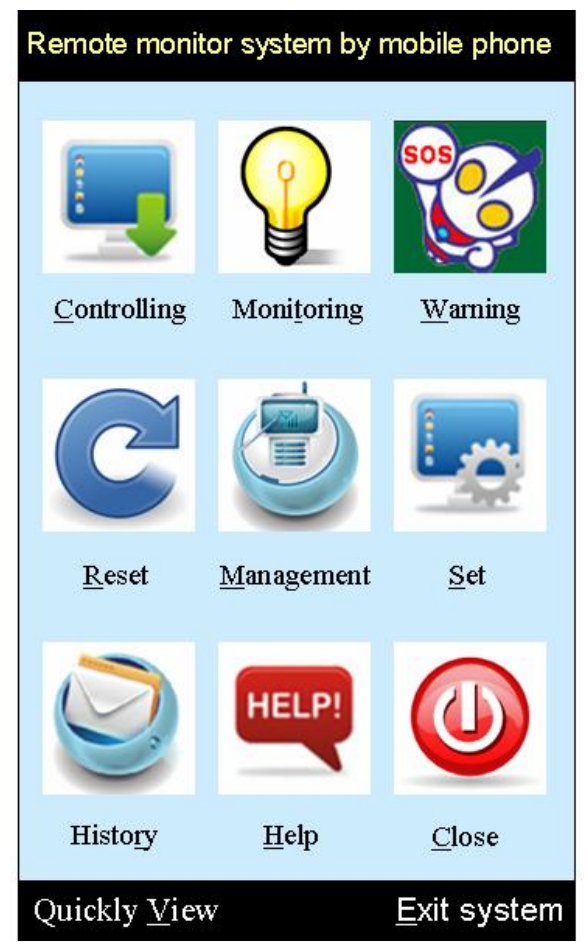

Fig.2. The user interface of the system

In Figure 2, the commonly used functions were placed on the top window. The function of each button is described as follows: clicking "monitor" button, the window will be popped up with the connection status and the current state of the controlling port of the hardware execution units (such as whether to activate the smoke sensor connection and sensor sent smoke density value). At the same time, the information will be reflected on the subsequent pop-up sub-menu with information of four channel analog signalsand four channel digital signals; once "Control" button is clicked, the system would check the connection of the hardware execution units under the "Monitor" firstly or it would start the "Monitor" button automatically. The user only can operate the sub-menu connected the hardware; the "Manage" button is to set the user's authority, and to modify the user's personal information. The function of "reset" button is to set the state of the hardware unit to the initial default state; "Alarm" button is to set trigger value of the separate ways monitor signal, corresponding alarm unit interface will pop up automatically with voice alarms, when the system alarm information. Clicking on the "history" button, we can browse the historical operating information. Other buttons will not be described here. 


\section{$B$. The compilation of the SMS operation code}

(1) Send messages: calling to the API provided by the system directly the messages can be sent. The key code is as follows:

Sms Manager sms = Sms Manager. getDefault(); sms. Send Text Message (phonenumber, null, msg, null, null);

//Send a message to a specified phone number

(2) Intercepted SMS: In order to distinguish between the ordinary SMS and the message from monitoring system, we need to take a certain measures to intercept text messages received. The procedure is shown in Figure 3.

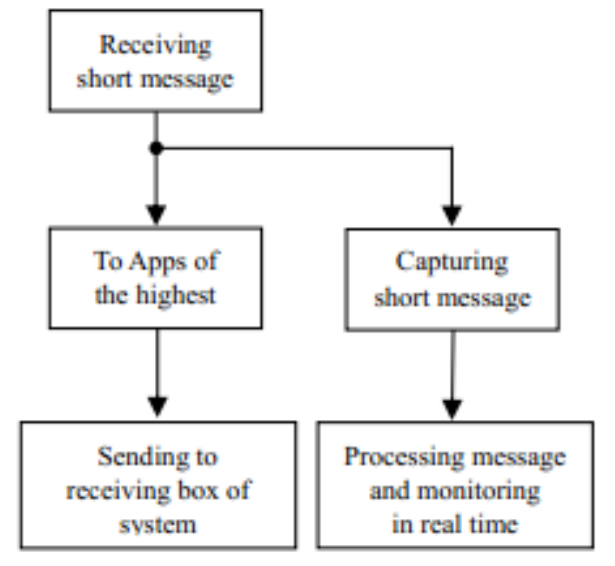

Fig.3. Intercept SMS procedure graph

The Android system needs to register a SMS listener for intercepting messages. After the system receives the message, the system would notice the listener of the highest priority first, until all listeners received or been canceled broadcasting [11]. It should be noted that, according to the official document released by Google, we can change the priority by modifying the priority parameter in the Manifest.xml. The value of parameter is from -1000 to 1000 , the higher the value, the higher the priority. Unfortunately, we find that, even if the parameter is set the maximum (1000), some anti-virus soft wares intercept the SMS earlier than the former.

What does it cause? How can the software intercept the message before the anti-virus software? Android system has an integer variable to hold the listener priority. Therefore, that setting the priority to the maximum value (2147483647) of the integer variable can make it proper. What's more, the priority of dynamic registration is higher than Static registered, so we chose the dynamically register priority with integer maximum. The key code is as follows:

IntentFilter filter $=$ new IntentFilter () ; filter.addAction("android.provider.Telephony.SMS_REC EIVED");

filter.setPriority(Integer.MAX_VALUE);

registerReceiver(new SmsReceiver(), filter); I/Dynamic registration
(3) filter SMS: Remote monitoring only needs to intercept messages sent by a fixed number. In order to allow the user to use the SMS function, the easiest way is to make a number matching. Firstly, let the user put its phone number into android database. Then, look for the number of received SMS in database, and if they are matched, the SMS will be intercepted, otherwise nothing will be done. Although we can take white list or do a signal analysis of the received SMS. If the SMS contained critical information, it would be intercepted. However, the instantaneity is not as good as the former.

\section{The hardware debugging and connection between UI and SMS Operator \\ SMS is the bridge between Android and hardware.} Developers can define their own communication rules. Just making sure that you send and receive SMS having no problem, the joint debjugging can be successful. The block diagram of Hardware and software debugging is shown in Figure 4.

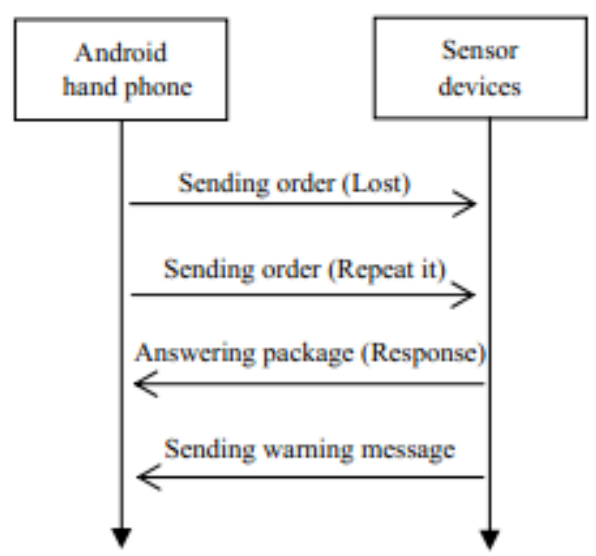

Fig.4. Hardware and software communication diagram

Safety and reliability is the great concern to the intelligent home. The remote monitoring system would have no market value because of negligence of security. Although the device is able to be controlled only by users' mobile phones, there is still risk in security if the phone was lost or stolen. So the password must be checked both starting and involving the privacy. Because of encryption, others can not know about what the user wants to do by SMS. Generally speaking, communication rules got hacked, the password authentication is useless, and others can send short messages to control the hardware directly. In addition, the receiver can improve system reliability by replying to encrypted confirmation SMS, but it is at the expense of real time.

\section{IV . Conclusion}

There are many ways to communicate between Android phones and hardware device, such as short message and wireless IP. The instantaneity is very good based on IP, but the UDP packet may be lost or TCP can't be connected etc in some place where the signal is not strong enough. To some 
extent, the requirement of signal is not so strict via short message. What we need to do is to send out the instruction to control the system, so you'd better use TCP/IP communications in the intranet.

In addition to the field of intelligent home, wireless monitor system has been applied to many other areas, and the space of development is extremely wide. With the increasing of internet speed and the acceleration of information transmission rate, it is not impossible to monitor any corner at any time and even to control everything in the near future. Besides, the Internet of Things will undergo a period of rapid development [12].

\section{References}

[1] Ming Ma, "Wireless network application status and development trend analysis," China science and technology review, 2012(32).

[2] Chunyi Tan and Feng Gao, "The on-board wireless anti-theft system based on GSM," Journal of JiangNan university (natural science edition), 2005, 4 (4): 386-389.

[3] Yong Gan, Xinxin Liu and Chunli Jia, "The remote monitoring anti-theft system based on ZigBee and TC35i design," Journal of ZhengZhou institute of light industry (natural science edition), 2012 (1).
[4] Ningqiang Bi and Ruixiang Zhu, "Based on tc35i GSM remote monitoring the soil information system," Agricultural mechanization research, 2012 (3).

[5] Xiuling He and Yue Li, "Wireless network based on Zigbee technology coal application," Coal mine machinery, 2012 (6).

[6] Xixiu Liu, Min Zhang and Yong Liu, "SM technology in the application of remote monitoring system," nstrument technique and sensor, 2012 (10).

[7] Hongzhen $\mathrm{Yu}$ and Dingxin He, "Household appliances remote control system based on GSM SMS," Automation and information engineering, 2006, 27 (2) : 24-25 to 40.

[8] Binwei Deng and Shian Yu, "The intelligent control system based on GSM module," Electronic technology in Shanxi, 2012 (4) : 33-35.

[9] Jiwei Zhang, "The intelligent home control system based on TC35," Electronic testing, 2012 (7) : 81-85.

[10] Peiming Fang, "New sensor principle and application," Electronic industry press, 2006

[11] Zhihong Guo, "Application development explanation of Android (Version 1)," Electronic industry press. 2010.

[12] Guojing Zeng, Yue Song and Zhihui He, "An intelligent home remote control system hardware design," Application of electronic technique, 2011, 37(4) 